\title{
Enhanced Recommendations for e-Learning Authoring Tools based on a Proactive Context-aware Recommender
}

\author{
Daniel Gallego, Enrique Barra, Aldo Gordillo and Gabriel Huecas \\ Escuela Técnica Superior de Ingenieros de Telecomunicación \\ Universidad Politécnica de Madrid, Avenida Complutense 30, 28040, Madrid, Spain \\ Email: \{dgallego,ebarra, agordillo, gabriel\}@ dit.upm.es
}

\begin{abstract}
Authoring tools are powerful systems in the area of e-Learning that make easier for teachers to create new learning objects by reusing or editing existing educational resources coming from learning repositories or content providers. However, due to the overwhelming number of resources these tools can access, sometimes it is difficult for teachers to find the most suitable resources taking into account their needs in terms of content (e.g. topic) or pedagogical aspects (e.g. target level associated to their students). Recommender systems can take an important role trying to mitigate this problem. In this paper we propose a new model to generate proactive context-aware recommendations on resources during the creation process of a new learning object that a teacher carries out by using an authoring tool. The common use cases covered by the model for having recommendations in online authoring tools and details about the recommender model itself are presented.
\end{abstract}

Index Terms-Authoring tools; Recommender systems; Learning objects; Proactivity; Context-awareness

\section{INTRODUCTION}

In the area of e-Learning, authoring tools can help educators to repurpose digitized resources or create complex Learning Objects (LOs) using existing contents from third-party learning repositories. These tools usually present an overwhelming variety of resources, causing a problem related to identifying what are the best ones considering the personal needs of the teacher.

This issue is mitigated in other areas of Technology Enhanced Learning (TEL) by using recommender systems [1]. But in the authoring tool field the use of such kind of systems has not been exploited yet. Authoring tools usually do not take into account the teacher's background and current context while the $\mathrm{LO}$ creation process is carried out. The recommender should take into account the history of the user in the current process so as to recommend similar resources. For these reasons, the application of a context-aware recommender system [2] could improve the teacher experience because sometimes simple suggestions following a user-request pattern are not enough when teachers do not know exactly what type of resources are available or when it is possible to request them in the creation process.

In this scenario, a proactive recommender system can play an important role in the decision of which educational contents are more appropriate for a given situation. By analyzing context-awareness information related to the user's needs, like the topic of the LO (i.e. physics) or the target audience (i.e. students level for a school teacher), the system could suggest suitable resources without explicit user request being needed. Therefore, the user would discover the resources at the same time as the requirements appear during the creation process.

Bearing in mind the previous scenario, in this paper we present a new general model to improve previous work on proactive context-aware recommendations in e-Learning systems [3] to be applied in online e-Learning authoring tools. It covers several use cases related to common situations involved in the use of authoring tools to create new LOs based on existing educational resources.

\section{RELATED WORK}

E-Learning authoring tools are computer based tools that allow a general group (habitually teachers) to create (or author) educational content and LOs that can be usually integrated in a Learning Management System [4].

In TEL a great variety of research and practical applications exist in the area of recommender systems [1]. They are not used only in LO repositories but also in other TEL environments like learning networks and teaching communities [5] or personal learning environments [6]. In addition, they are not exclusively used to recommend interesting LOs but other users and activities [7].

Finally, as Verbet et al. show in their recent survey [2], the use of context-aware recommender systems is becoming relevant in the area of e-Learning. These systems take advantage of contextual information [8] about the user and his/her circumstances so as to increase the level of personalization in the suggestions provided. One specific research line that is getting very popular associated to utilize context is the introduction of proactivity, giving rise to proactive context-aware recommender systems [9]. These systems push recommendations to the user when the current situation seems appropriate, without explicit user request, going beyond traditional recommender systems. [10] propose a proactive recommender system in computer-supported learning that works on LO repositories and adapts to a student's profile. Whereas in [3] we propose a proactive context-aware recommender system capable of recommending both, LOs and 
similar peers in an e-Learning social network based on social, location and user context-awareness information.

\section{A Model For Proactive Context-Aware RECOMMENDATIONS IN AUTHORING TOOLS}

Fig. 1 presents the general model to incorporate proactive recommendations into e-Learning authoring tools. It covers the following use cases that appears attending to its temporary nature from the point of view of a user utilizing the authoring tool in a general scenario: 1) Recommending when the user is starting the creation process, 2) Recommending while the user is creating the learning object and 3) Recommending novel resources when reviewing or editing learning objects.

\section{A. Context}

Using context-awareness information is now a common feature to improve recommender systems in e-Learning on the basis that the more information you have from a user, the more personalized and accurate the results provided will be [2].

We understand context as any information that can be used to characterize the situation of an entity [8]. Therefore, our system is based on two context categories to provide proactive recommendations on relevant educational content related to the LO creation process the teacher carries out: User context, i.e. the current activity of the user during the creation process in the authoring tool (e.g. idle or checking educational content like a video); and Educational context, i.e. the information related to the educational resources the teacher is using for creating a new LO (e.g. topic, language or target age).

\section{B. Initial Input}

The model begins with an optional step consisting of providing initial input about the main educational context values related to the $\mathrm{LO}$ the teacher wants to create, like the general topic (e.g. biology), the target level of his/her students (e.g. 14-15 years old), the language (e.g. English) or the target device where the LO will be consumed (e.g. mobile device or desktop computer). Having such kind of data since the beginning would help the system to focus the recommendations sooner, achieving this way the use case 1.

This initial input also covers the use case 3 presented above. When a teacher reviews a LO previously created or edits a LO in a draft state, all the resources used on it are considered to generate an initial educational context input. This allows the recommender to look for novel resources in the same area that did not exist or maybe were not considered relevant in the past.

\section{Phase I: Resource Profiling}

This is the first step of the 3-phase loop illustrated in Fig. 1. It is in charge of gathering all the metadata related to the resources used during the creation process in order to answer the question What kind of content have been used? This set of metadata conforms the educational context of the LO the teacher is creating. Therefore, the more information the system knows about the resources the teacher is using, the more accurate would be the recommendations provided in the third phase. As a result, the educational context is generated and sent to the next phases.

\section{Phase II: Situation Assessment}

The second phase tries to answer the question When to make a recommendation?. To do it the system calculates a score $\mathrm{S} 1$ which is a number between 0 and 1 . If $\mathrm{S} 1$ exceeds a threshold $\mathrm{T} 1$, the third phase will be initiated. If $\mathrm{S} 1=1$, the highest possible value, then a recommendation would be triggered in any case. If the current situation does not warrant a recommendation, no matter how high a particular resource score, $\mathrm{S} 1$ is set to 0 and the recommendation process is aborted without considering resources to recommend. Note that this phase does not take properties of resources into account (i.e. the educational context previously generated). However it considers the current user context represented by the teacher's activity in the authoring tool. For instance, a situation would be more appropriate for a proactive recommendation if the user is idle or browsing resources in the system, compared to a situation in which the teacher is viewing a resource (e.g. a video) to decide if it is suitable or not. This appropriateness factor can be derived by the level of interruptibility allowed by the users in every situation involved in proactive systems. As Gallego et al. [9] shown, the system should avoid disturbing the user if he/she is focused on other important task.

That contextual information related to the user is needed as a prerequisite to calculate $S 1$. In our model, the user context is provided by the platform through the connections between the authoring tool and the recommender system. The parameters related to this user context are domain dependent, and have to be studied for each specific scenario.

Furthermore, the score S1 has an impact on the threshold $\mathrm{T} 2$ of the third phase, i.e. the higher $\mathrm{S} 1$ is, the lower $\mathrm{T} 2$ is set. Therefore, the threshold $\mathrm{T} 2$ is a function of $\mathrm{S} 1$ in the form: $T 2=|1-S 1|$.

\section{E. Phase III: Resource Assessment}

The third phase evaluates the suitability of particular resources trying to answer the question Which resources to recommend?. Any recommender algorithm that considers the educational context information provided is valid. A content-based [11] would be a good option as it recommends items that are similar to those that a user has utilized or liked in the past, or as in our case, has selected in the current LO creation process. Hence, various candidate resources would be compared with other previously used by the user and the best-matching resources are recommended. This can be improved by adding the information store in the recommender data base that allows the system to increase the weight of those resources that have been used intensively by other teachers, meaning their relevancy in that area of knowledge.

The result must be a score $\mathrm{S} 2$ normalized to [0,1] (with $\mathrm{S} 2=1$ being the best possible score) for each resource in the candidate set. S2 corresponds to the predicted rating of the recommendation algorithm selected. The candidate resources 


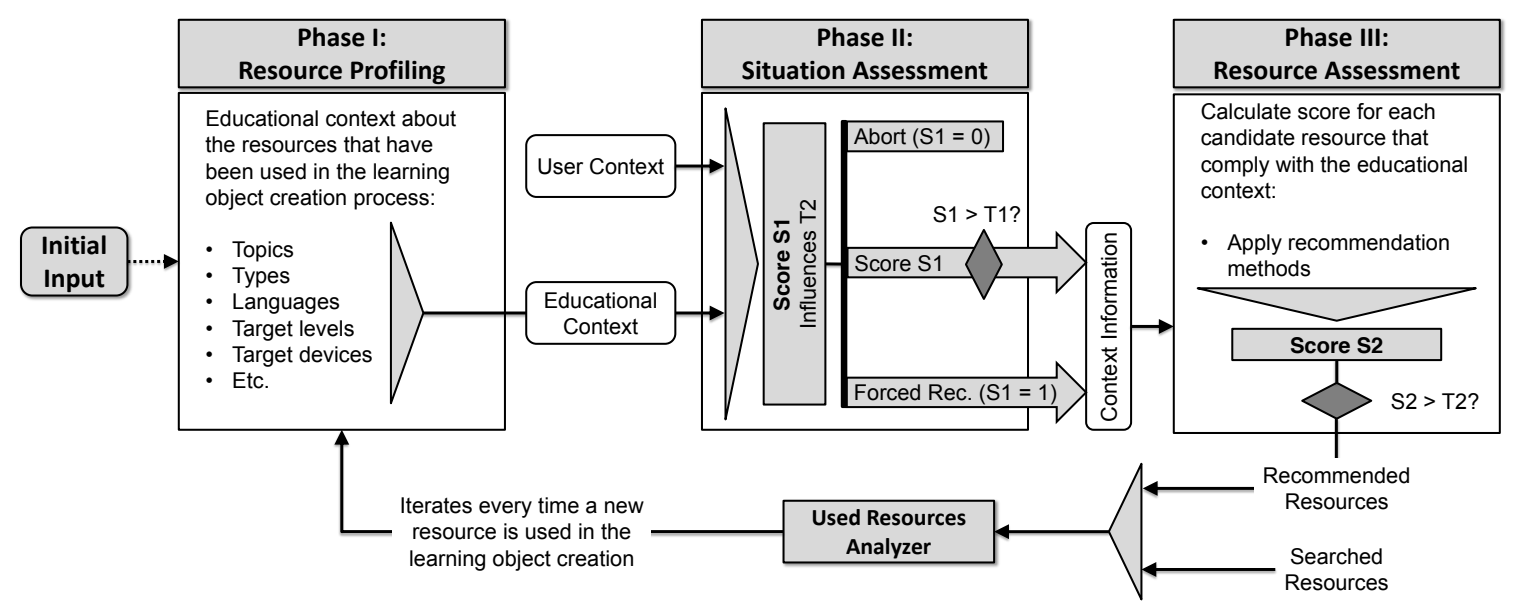

Fig. 1. Model for generating proactive context-aware recommendations in e-Learning authoring tools.

would be ranked according to S2 and tested against the threshold $\mathrm{T} 2$. If $\mathrm{S} 2>\mathrm{T} 2$ for a resource, then it is finally considered for recommendation and the user is notified. If no resource score S2 exceeds the threshold $\mathrm{T} 2$, then no one is recommended, the process is aborted and restarted when the condition to loop again is met.

After the recommended items are communicated to the teacher, he/she might select one of them or might not (i.e. by accepting or rejecting the recommendation). This occurs also with the resources the teacher looks for in the authoring tool (as they can be suitable or not for his/her interests). In Fig. 1 this is represented by the loop linking the last phase with the first one. It takes into account all the resources (i.e. recommended or searched) to be evaluated by the used resources analyzer. Each time a new resource is added to the LO the teacher is creating, the analyzer fires a new loop that initiates the model again (as new resources information is available for the first phase). As a result, the use case 2 is achieved because the loop is continually repeated while the previous condition is reached.

\section{CONClusion And Future Work}

In this paper we have presented a novel model for generating proactive context-aware recommendations in e-Learning authoring tools. It is based on analyzing the current user activity as well as the educational context related to the resources the teacher selects during the learning object creation process to recommend similar resources. This is done in several temporal instants: at the beginning of the creation process, during the process itself and when the learning object is reviewed or edited to be improved with novel material after it has been created. The recommendations are provided in a proactive way when the situation seems appropriate without explicit user request.

The model has been designed to support a general scenario in which no previous information about the user is needed, being the only requirement the necessity of having connection to online general content providers and educational learning repositories.

Open challenges consists of implementing the model in a real scenario related to an existing online e-Learning authoring tool in order to validate it. In relation with this, studying the impact in the user experience of teachers having such kind of proactive recommendation in an authoring tool would be an interesting research line to investigate.

\section{REFERENCES}

[1] N. Manouselis, H. Drachsler, R. Vuorikari, H. Hummel, and R. Koper, "Recommender systems in technology enhanced learning," in Recommender Systems Handbook, F. Ricci, L. Rokach, B. Shapira, and P. B. Kantor, Eds. Springer US, 2011, pp. 387-415.

[2] K. Verbert, N. Manouselis, X. Ochoa, M. Wolpers, H. Drachsler, I. Bosnic, and E. Duval, "Context-aware recommender systems for learning: A survey and future challenges," Learning Technologies, IEEE Transactions on, vol. 5, no. 4, pp. 318-335, 2012.

[3] D. Gallego, E. Barra, S. Aguirre, and G. Huecas, "A model for generating proactive context-aware recommendations in e-learning systems," in Frontiers in Education Conference, 2012, pp. 1364-1369.

[4] T. Murray, S. Blessing, and S. Ainsworth, Authoring Tools for Advanced Technology Learning Environments: Toward Cost-Effective Adaptive, Interactive and Intelligent Educational Software. Kluwer Academic Publishers, 2003.

[5] F. Soude, H. Drachsler, F. Brouns, and P. Sloep, "A trust-based social recommender for teachers," in Proceedings of the Workshop on Recommender Systems for Technology Enhanced Learning, 2012, pp. 49-60.

[6] F. Mödritscher, "Towards a recommender strategy for personal learning environments," Procedia Computer Science, vol. 1, no. 2, pp. $2775-$ 2782, 2010.

[7] K. Verbert, H. Drachsler, N. Manouselis, M. Wolpers, R. Vuorikari, and E. Duval, "Dataset-driven research for improving recommender systems for learning," in Proceedings of the 1st International Conference on Learning Analytics and Knowledge. ACM, 2011, pp. 44-53.

[8] A. K. Dey, "Understanding and using context," Personal Ubiquitous Comput., vol. 5, no. 1, pp. 4-7, Jan. 2001.

[9] D. Gallego, W. Woerndl, and G. Huecas, "Evaluating the impact of proactivity in the user experience of a context-aware restaurant recommender for android smartphones," Journal of Systems Architecture.

[10] A. Ruiz-Iniesta, G. Jimenez-Diaz, and M. Gomez-Albarran, "Recommendation in repositories of learning objects: A proactive approach that exploits diversity and navigation-by-proposing," in Ninth IEEE International Conference on Advanced Learning Technologies, ICALT 2009, 2009, pp. 543-545.

[11] M. J. Pazzani and D. Billsus, "The adaptive web," P. Brusilovsky, A. Kobsa, and W. Nejdl, Eds. Berlin, Heidelberg: Springer-Verlag, 2007, ch. Content-based recommendation systems, pp. 325-341. 